\title{
Circulating Pre-microRNA-488 in Peripheral Blood Is a Potential Biomarker for Predicting Recurrence in Breast Cancer
}

\author{
TAKAAKI MASUDA ${ }^{1}$, YOSHIAKI SHINDEN ${ }^{1}$, MIWA NODA ${ }^{1}$, HIROKI UEO ${ }^{1,2}$, \\ QINGJIANG HU ${ }^{1}$, YUKIHIRO YOSHIKAWA ${ }^{1}$, YUSUKE TSURUDA ${ }^{1}$, YOSUKE KURODA ${ }^{1}$, \\ SHUHEI ITO $^{1}$, HIDETOSHI EGUCHI ${ }^{1}$, SHINJI OHNO ${ }^{3}$ and KOSHI MIMORI ${ }^{1}$ \\ ${ }^{1}$ Department of Surgery, Kyushu University Beppu Hospital, Beppu, Japan; \\ ${ }^{2}$ Department of Breast Surgery, Saiseikai Fukuoka General Hospital, Fukuoka, Japan; \\ ${ }^{3}$ Breast Oncology Center, The Cancer Institute Hospital Ariake of \\ Japanese Foundation for Cancer Research, Tokyo, Japan
}

\begin{abstract}
Background/Aim: Circulating microRNAs (miRs) in blood have been highlighted as diagnostic, prognostic and predictive biomarkers for "Precision medicine". This study aimed to explore the possibility of using circulating precursor miRs (pre-miRs) as clinical biomarkers of recurrence in breast cancer. Materials and Methods: We performed miR microarray analyses of circulating miRs in blood from patients with or without recurrence of breast cancer and identified miR-488-5p as a recurrence-related $m i R$. Then, the expression levels of pre-miR-488 or miR-488$5 p$ were measured by $R T-q P C R$ and the clinicopathological and prognostic significance of circulating pre-miR-488 was assessed in the blood of breast cancer patients. Results: A positive correlation was noted between pre-miR-488 and miR-488-5p expression in blood. In 330 cases of surgicallytreated breast cancer, high expression of circulating pre-miR488 was an independent poor prognostic factor for recurrence-free survival. Conclusion: Circulating pre-miR488 expression could be a novel prognostic biomarker for predicting recurrence in breast cancer patients.
\end{abstract}

"Precision medicine" is defined as personalized treatment of patients with similar clinical symptoms on the basis of differences in genetics, biomarkers or phenotypes. It is expected to improve the prognosis of various malignancies, including breast cancer (1). There is growing interest in the

Correspondence to: Koshi Mimori, MD, Ph.D., Department of Surgery, Kyushu University Beppu Hospital, 4546 Tsurumihara, Beppu 874-0838, Japan. Tel: +81 977271650, Fax: +81 977271651, e-mail:kmimori@beppu.kyushu-u.ac.jp

Key Words: Pre-miR-488, breast cancer, biomarker, liquid biopsy. use of "liquid biopsies" to identify biomarkers for "precision medicine" (2). "Liquid biopsy" is defined as the identification of biomarkers in body fluids, mainly in blood, and can provide non-invasive "real-time" information regarding tumor characteristics (2).

Recently, circulating microRNAs (miRs) in blood have been highlighted as diagnostic, prognostic and predictive biomarkers because of their high tissue specificity, stability and altered expression in tumor development (3). In particular, miRs in serum exosomes (actively secreted extracellular vesicles) are more stable than other forms, and they are expected to have potential applications as biomarkers $(4,5)$. Thus, miR analysis in liquid biopsies could offer a less invasive and more cost-effective biomarker for precision medicine (6).

miRs, short (17-22 bases) non-coding RNAs, regulate various cell functions, including proliferation, differentiation, cell fate determination and apoptosis. This is achieved by binding to the 3'-untranslated region of one or multiple target mRNAs followed by an inhibited expression (7). miRs play key roles in various aspects of tumor progression (8) as we reported elsewhere $(5,9,10)$. The biogenesis of the majority of miRs involves transcription by RNA polymerase II into primary miRs and subsequent processing by the nuclear enzyme Drosha into shorter hairpin structures, called pre-miRs. The pre-miRs are then transported into the cytoplasm where they are further processed into mature miRs by the enzyme Dicer (11).

Many reports have shown the clinical significance of mature miR expression in various cancers (8). However, the significance of pre-miR expression remains unclear. PremiRs, like mature miRs, are enveloped by exosomes and secreted into body fluids where they function as intercellular messengers $(12,13)$. We focused on the possible advantages 
of assessing pre-miRs rather than mature miRs, as detection is less costly and easier to conduct.

In this study, miR-488-5p was first identified as a recurrence-related exosomal $\mathrm{miR}$ and the clinicopathological and prognostic significance of pre-miR-488 expression was assessed in the blood of breast cancer patients. Furthermore, the correlation of pre-miR-488 and miR-488-5p expression in the blood of such patients was examined.

\section{Materials and Methods}

Clinical samples. In an effort to identify recurrence-related exosomal miRs in serum, 10 serum samples were obtained from breast cancer patients who had undergone surgery for a primary tumor. Surgery was conducted at the Department of Surgery, Kyushu University Beppu Hospital (Beppu, Japan) in 2013. Among the 10 samples, 5 were from patients with recurrence, and 5 were from those without recurrence. Serum samples were stored at $-80^{\circ} \mathrm{C}$ until miR extraction.

To determine the clinical significance of pre-miR-488 expression in the blood, we used updated clinical data files from breast cancer patients described elsewhere (14). Briefly, a total of 594 patients with breast cancer underwent resection of the primary tumor at the Department of Breast Oncology, National Kyushu Cancer Center (Fukuoka, Japan) from 2000 to 2008. Of these, 356 female patients with breast cancer without distant metastases, preoperative therapy or previous treatment for various cancers were included in this study. Among them, 330 were invasive ductal carcinomas (IDC), and 26 were ductal carcinomas in situ (DCIS). Survival analysis was performed for the 330 patients with IDC. The control samples were obtained from 11 healthy volunteers at Kyushu University Beppu Hospital. Aspiration of blood was conducted immediately before operation under general anesthesia. Blood was obtained through a venous catheter. The first $\mathrm{ml}$ of blood was discarded because of possible contamination by epidermal cells. Each $1 \mathrm{ml}$ sample of blood was immediately mixed with $4 \mathrm{ml}$ of ISOGEN-LS (Nippon Gene, Toyama, Japan) and stored at $-80^{\circ} \mathrm{C}$ until RNA extraction. Polymorphprep $^{\text {TM }}$ (Alere Technologies AS, Oslo, Norway) was used to separate 3 blood cell fractions (polymorphonuclear leukocytes (PMN), mononuclear cells (MC) and erythrocytes (RBC)). Samples were obtained from 9 patients with IDC who underwent primary tumor resection at Kyushu University Beppu Hospital in 2017. Each fraction was mixed with ISOGEN II (Nippon Gene, Toyama, Japan) and stored at $-80^{\circ} \mathrm{C}$ until RNA extraction.

Tumor tissues and paired normal tissues were obtained from 82 breast cancer patients who had undergone primary tumor resection at Kyushu University Beppu Hospital and affiliated hospitals between 1992 and 1996. The frozen tissue specimens were homogenized, mixed with ISOGEN (Nippon Gene) and stored at $80^{\circ} \mathrm{C}$ until RNA extraction. From this cohort, we used 30 highquality RNA samples for RT-qPCR analysis.

All patients gave written informed consent to participate in this study, which was approved by each institutional review board and the Ethics and Indications Committee of each institution. The observation periods ranged from 0.3 to 6.9 years (median 3.8 years). Postoperative adjuvant therapy was performed according to the St.Gallen Consensus Conference (15). The patients underwent clinical examinations at least every 3 months and annual mammographies and were further tested only if they had symptoms.
The stages and grades of the tumors were classified according to the AJCC/UICC TNM classification and stage groupings. All data for the samples, including age, pathological tumor size, nuclear grade, venous involvement, lymphatic involvement, lymph node metastasis, and the status of ER, PgR and HER2 were obtained from the medical records. The status of ER, PgR and HER2 was determined by immunohistochemical analysis. 0/1+ was defined as negative and $2+/ 3+$ as positive in the HER2 status of this study. Overall survival (OS) was defined as the time between the date of diagnosis and the date of death. Recurrence-free survival (RFS) was defined as the length of time after surgical treatment for cancer during which the patient survived without a sign of recurrence.

Isolation of exosomes in serum. Exosomes were isolated from serum by ultracentrifugation as described previously (5).

Extraction of total RNA. Extraction of total RNA from serum exosomes was performed using the miReasy serum/plasma kit (Qiagen, Venlo, Netherlands) according to the manufacturer's protocol. Frozen tissue specimens were homogenized, and total RNA was extracted with ISOGEN according to the manufacturer's instructions. Total RNA of blood was extracted according to the ISOGEN-LS manufacturer's instructions.

PCR cloning. Human pre-miR-488 and RNA18S5 were amplified by PCR with the primer pairs as follows: pre-miR-488, forward 5'CTCTCCCAGATAATGGCACTCTCA-3' and reverse 5'-AGAGTC ATCTGACCAAGAAATAGCC-3'; RNA18S5, forward 5'-AGTCCC TGCCCTTTGTACACA-3' and reverse 5'-CGATCCGAGGG CCTCACTA-3'. The PCR products were cloned into a pCR2.1 cloning vector (Invitrogen, Carlsbad, CA) according to the manufacturer's instructions. We used each plasmid as a reference for calibration of the expression level of each target gene (pre-miR-488 or RNA18S5 at quantitative PCR (qPCR) as described below.

RT-qPCR Assay. RT-qPCR assessments of pre-miR-488, RNA18S5 and $G A P D H$ were performed as previously described (5). In brief, RT was performed with random hexamers using M-MLV reverse transcriptase (Invitrogen, Carlsbad, CA, USA). qPCR was performed with LightCycler ${ }^{\circledR}$ FastStart DNA Master SYBR Green I (Roche, Basel, Switzerland). The raw data are presented as the relative quantity of target genes, normalized with respect to RNA18S5 or $G A P D H$. The primer sequences for RT-PCR were as follows: premiR-488, forward 5'- CTCTCCCAGATAATGGCACTCTCA-3' and reverse 5'- AGAGTCATCTGACCAAGAAATAGCC-3'; RNA18S5, forward 5'- AGTCCCTGCCCTTTGTACACA-3' and reverse 5'CGATCCGAGGGCCTCACTA-3' and $G A P D H$, forward, 5'TTGGTATCGTGGAAGGACTC-3' and reverse 5'-AGTAGAGG CAGGGATGATGT-3'.

RT-qPCR assessments of $m i R-488-5 p$ and $R N U 6 B$ were performed as described previously (5). In brief, $m i R-488-5 p$ and $R N U 6 B$-specific cDNAs were synthesized using gene-specific primer sets according to the TaqMan ${ }^{\circledR}$ Micro-RNA Reverse Transcription Kit protocol (Applied Biosystems, Foster City, CA, USA). qPCR was performed using PCR LightCycler ${ }^{\circledR} 480$ Probes Master (Applied Biosystems). $m i R-488-5 p$ and $R N U 6 B$ were purchased from Applied Biosystems.

Relative quantification of miR expression was calculated using the $2^{-\Delta \Delta C t}$ method. The raw data are presented as the relative quantity of each cloned plasmid or cDNA from Human Universal Reference 


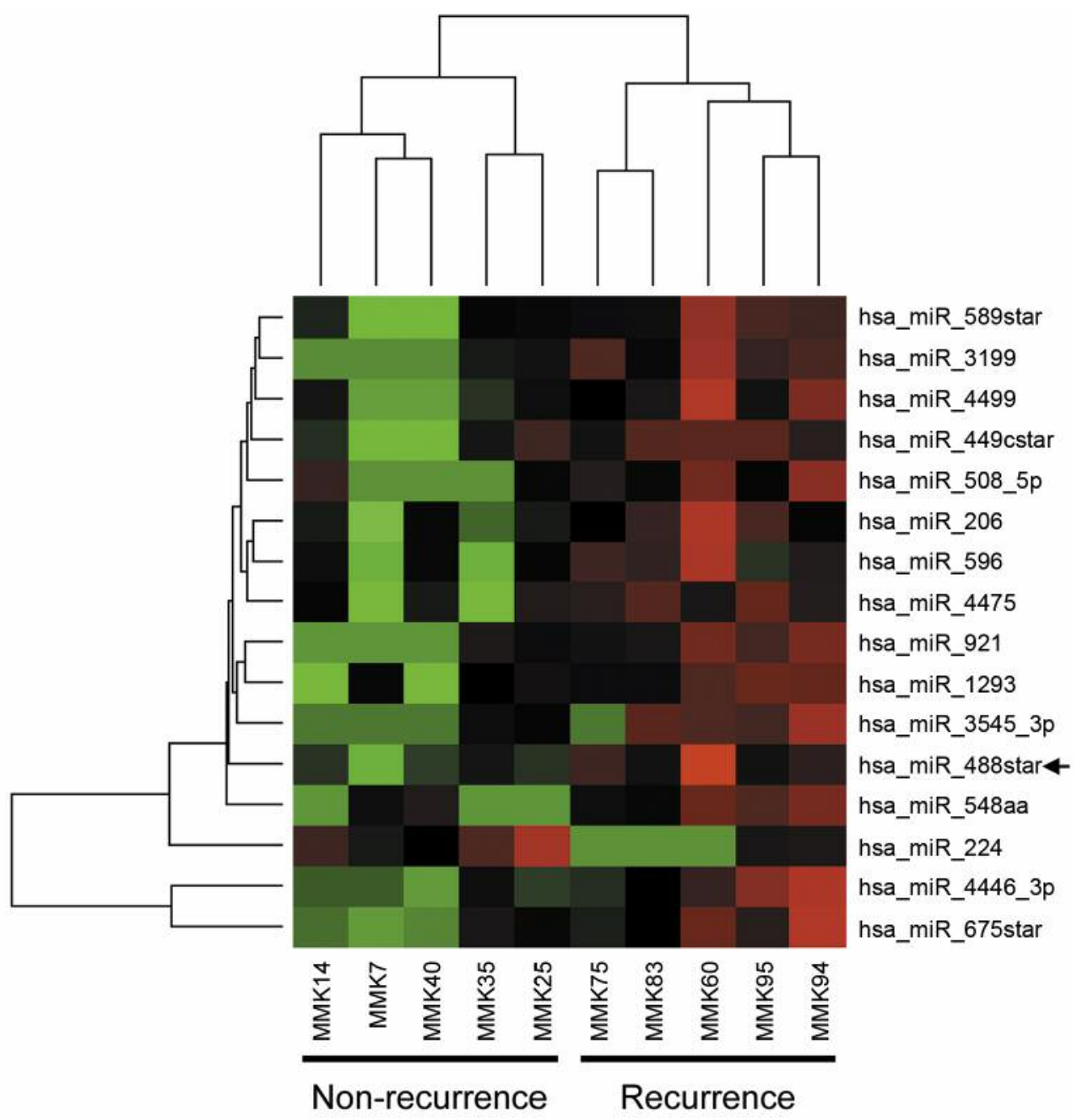

Figure 1. Heatmap of exosomal miR profiles in serum from breast cancer patients with and without recurrence. The expression levels of these miRs range from low (green) to high (red). Patients 75, 83, 60, 95 and 94 had recurrence and patients 14, 7, 40, 35 and 25 had no recurrence.

Total RNA (Clontech Laboratories, Palo Alto, CA, USA) and normalized by internal control to RNA18S5, GAPDH or RNU6B. The PCR product was electrophoresed with $3 \%$ agarose gel and stained with ethidium bromide.

miR microarray analysis. We performed miR microarray as described previously (10). In brief, extracted total RNA was labeled with Hy5 using the miRCURY LNA microRNA Array labeling kit (Exiqon, Vedbaek, Denmark). Labeled RNAs were hybridized onto 3D-Gene Human miRNA Oligo chips 17v 1.00 (Toray, Kamakura, Japan). Fluorescent signals were scanned and analyzed using the 3D-Gene Scanner (Toray). The raw data from each spot were normalized by subtraction of the background signal mean intensity, determined by the $95 \%$ confidence intervals of the signal intensities of all blank spots. Valid measurements were considered as those in which the signal intensity of both duplicate spots was $>2$ s.d. of the background signal intensity. Cluster analysis was performed to identify exosomal miRs in serum that were statistically differentially expressed between breast cancer patients with and without recurrence $(p<0.05$, Student's $t$-test $)$. The heatmap of the miRs profile is illustrated in Figure 1. The expression levels of these miRNAs range from low (green) to high (red). Patients 75, 83, 60, 95 and 94 had recurrence and patients $14,7,40,35$ and 25 had no recurrence.

Statistical analysis. For clinical analysis, cases were divided into two groups using the minimum $p$-value approach based on the miR-488 expression level, which is a comprehensive method to identify the optimal risk separation cutoff point in continuous gene expression measurements for survival analysis in multiple datasets (16). Associations between the variables were tested with the MannWhitney $U$-test, Student's $t$-test or Fisher's exact test. The degree of linearity was estimated by Spearman's rank correlation co-efficient. Survival curves were drawn according to the Kaplan-Meier method, and survival analysis was carried out by the log-rank test when two curves were being compared. Cox proportional hazards regression 
A

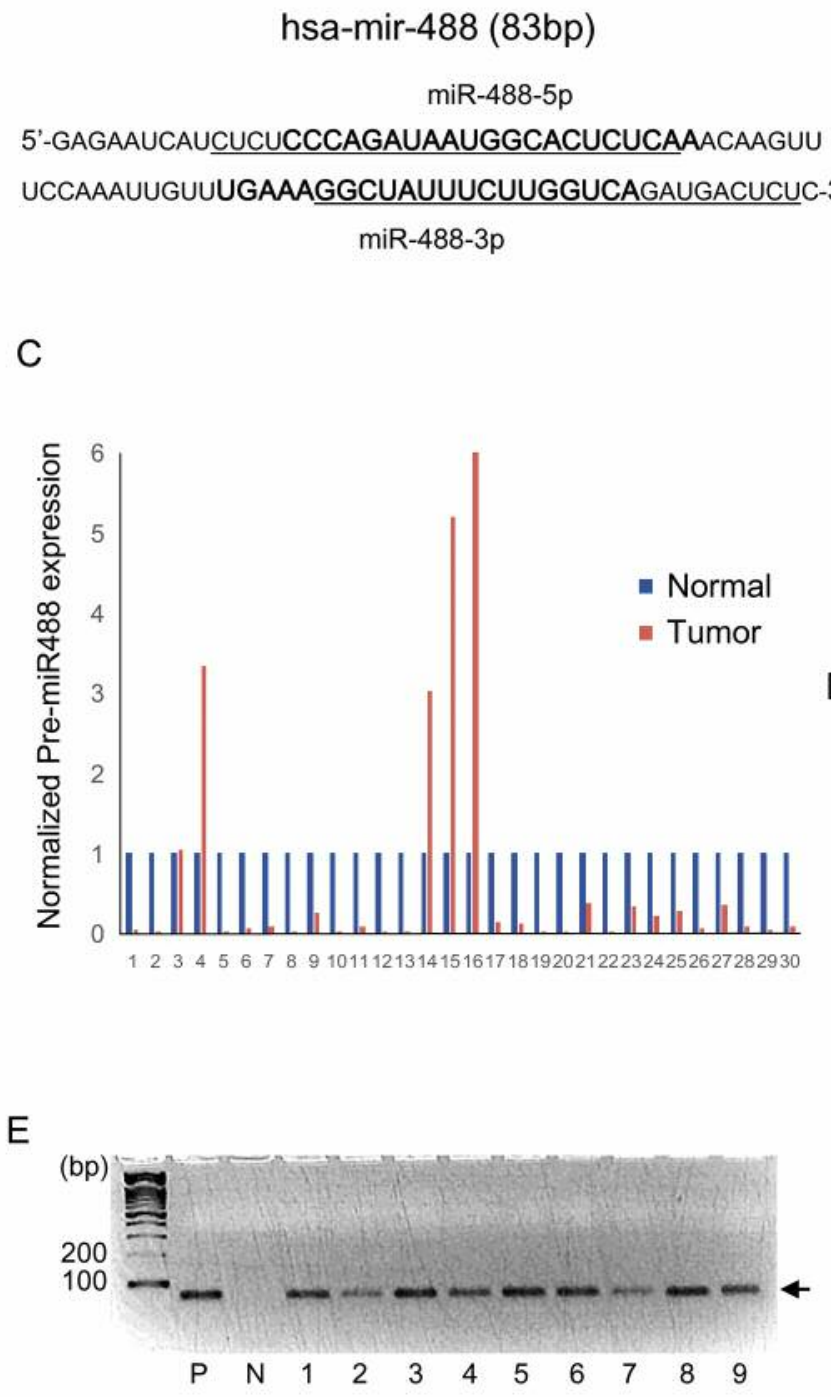

B

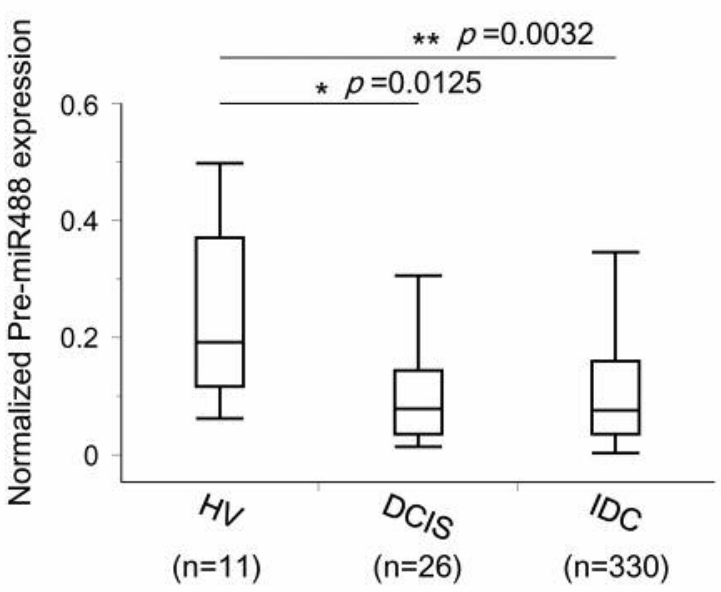

D

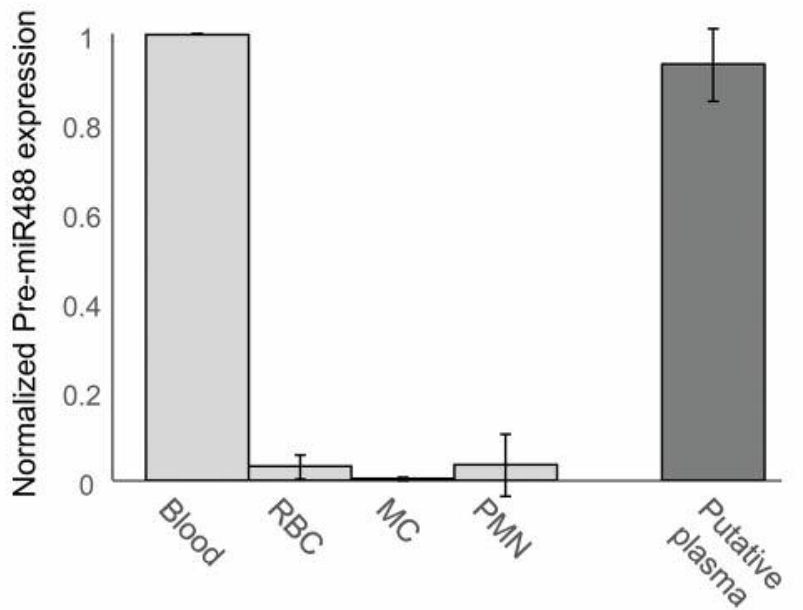

$\mathrm{F}$

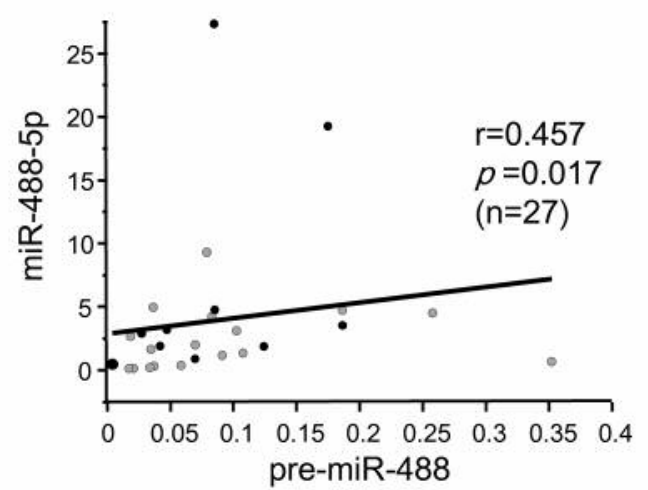

Figure 2. Pre-miR-488 expression in breast cancer. A. The sequence of human pre-miR-488. The sequences in boldface are miR-488-5p and miR-488-3p. Underlined sequences are paired primers for PCR to detect pre-miR-488. B. Pre-miR-488 expression in blood samples from breast cancer patients. C. Pre-miR-488 expression in tissues of breast cancer patients. red: tumor tissues; blue: normal tissues. D. Pre-miR-488 expression in 3 blood fractions of breast cancer patients. The expression level in plasma is defined as follows: [putative plasma]=[whole blood]-[PMN]-[PBMC]-[RED]. E. Pre-miR-488 expression in the plasma of breast cancer patients. F. Correlation between pre-miR-488 and miR-488-5p expression in blood of breast cancer patients. $r=0.457, p=0.017$, Spearman's rank correlation co-efficient. 
A

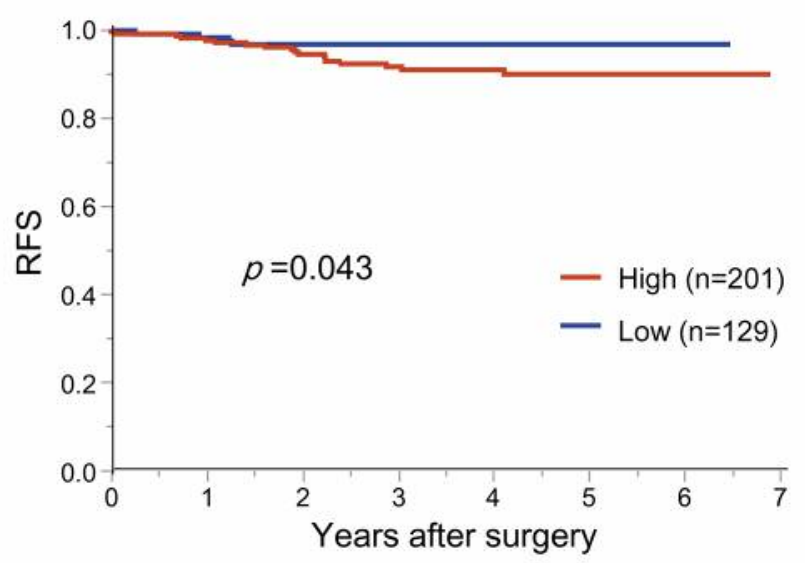

B

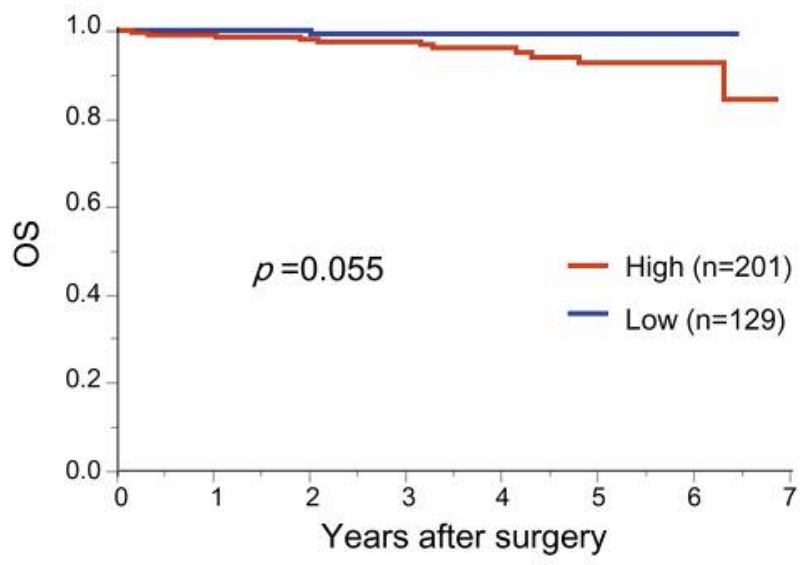

C

HR+/HER2-

HR $\pm / H E R 2+$

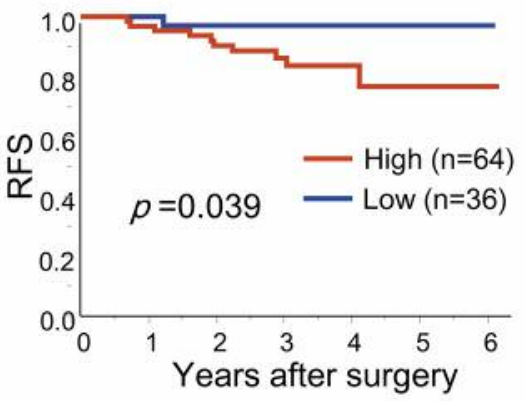

TN

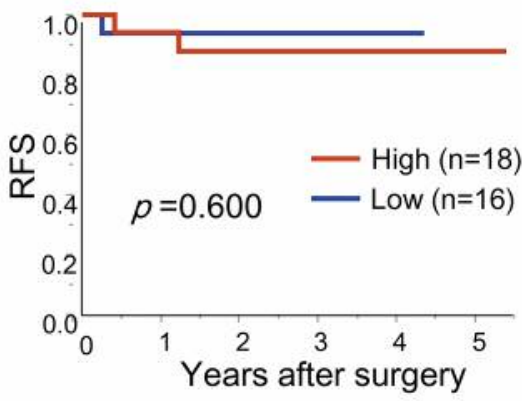

Figure 3. Kaplan-Meier survival curve of breast cancer patients according to pre-miR-488 expression level to in blood. A. RFS. B. OS. C. Subgroup analysis for RFS of patients according to the status of HR and HER2. HR: Hormone receptor; TN: triple (ER, PgR, HER2) negative.

was used to determine univariate and multivariate hazard ratios for survival. A two-sided $p<0.05$ was deemed statistically significant. Statistical analyses were performed using JMP Pro 13 software (SAS Institute, Cary, NC, USA).

\section{Results}

miR profiling and identification of recurrence-related exosomal miRs in serum. It is well recognized that circulating miRs in blood are mainly carried by exosomes $(8,9)$. To identify recurrence-related exosomal miRs in the serum of breast cancer patients, miR microarray analyses of exosomal miRs was performed from 5 patients with recurrence and 5 patients without recurrence. The heatmap of the miR profile is illustrated in Figure 1. The expression levels of these miRNAs ranged from low (green) to high (red). Various miRs were significantly down-regulated or upregulated in the cases with recurrence compared to those without recurrence. Among them, we focused on miR-488-
$5 \mathrm{p}$ as an up-regulated $\mathrm{miR}$ in the cases with recurrence because its clinical significance is unknown in breast cancer.

Pre-miR-488 expression in blood or breast cancer tissues of breast cancer patients. The findings shown in Figure 1 indicated that it would be useful to characterize the clinical significance of pre-miR-488 expression as well as its utility as a clinical biomarker in the blood of breast cancer patients. The sequence of pre-miR-488 is shown in Figure 2A. The underlined regions are paired primers for the PCR. The expression levels of circulating pre-miR-488 from 330 patients with IDC, 26 patients with DCIS and 11 healthy controls are plotted in Figure 2B. The pre-miR-488 levels of the control cases, DCIS cases, and IDC cases ranged from 0.061 to 0.496 (median, 0.191), from 0.012 to 0.328 (median, 0.078) and from 0.001 to 1.072 (median, 0.075), respectively. Interestingly, premiR-488 expression in the blood was significantly lower in patients with IDC as well as DCIS compared with healthy 
Table I. Univariate and multivariate analysis of prognostic factors for DFS of breast cancer patients.

\begin{tabular}{|c|c|c|c|c|c|}
\hline \multirow[t]{2}{*}{ Variables } & & \multicolumn{2}{|c|}{ Univariate analysis } & \multicolumn{2}{|c|}{ Multivariate analysis } \\
\hline & & $\mathrm{HR}(95 \% \mathrm{CI})$ & $p$-Value & HR $(95 \% \mathrm{CI})$ & $p$-Value \\
\hline Age (years) & $(\geq 65 /<65)$ & $0.58(0.14-1.71)$ & 0.350 & & \\
\hline Pathological tumor size & $(2,3 / 1)$ & $2.22(0.92-5.86)$ & 0.075 & $2.04(0.77-5.79)$ & 0.150 \\
\hline Nuclear grade & $(3 / 1,2)$ & $1.70(0.69-4.02)$ & 0.240 & & \\
\hline Venous involvement & $(+/-)$ & $1.862 \mathrm{e}-9(1.46-1.46)$ & 0.103 & & \\
\hline Lymphatic involvement & $(+/-)$ & $4.68(1.92-13.04)$ & 0.001 & & \\
\hline Lymph node metastasis & $(+/-)$ & $3.84(1.62-10.05)$ & 0.002 & $3.21(1.23-9.08)$ & 0.017 \\
\hline ER & $(+/-)$ & $0.48(0.21-1.16)$ & 0.098 & & \\
\hline PgR & $(+/-)$ & $0.39(0.15-0.91)$ & 0.028 & $0.55(0.20-1.45)$ & 0.230 \\
\hline HER2 & $(2,3 / 0,1)$ & $2.14(0.92-5.00)$ & 0.078 & $1.81(0.69-4.97)$ & 0.142 \\
\hline Pre-miR-488 & (high/low) & $2.91(1.09-10.09)$ & 0.032 & $3.19(1.11-11.60)$ & 0.030 \\
\hline
\end{tabular}

CI: Confidence interval.

controls. There was no significant difference in pre-miR-488 expression levels in blood between of DCIS and IDC. Next, pre-miR-488 expression was assessed in IDC tissues (Figure 2C). Pre-miR-488 expression in tumor tissues was lower than normal tissues in $83.3 \%$ of 30 breast cancer patients although in some cases, pre-miR-488 expression in tumor tissues was much higher than in normal tissues. These results may suggest that down-regulation of pre-miR-488 reflects carcinogenesis rather than tumor progression of breast cancer.

Assessment of pre-miR-488 in blood cell fractions and plasma. The distribution of pre-miR-488 in blood was assessed by RT-qPCR. Whole blood is composed of polymorphonuclear leukocytes (PMN), mononuclear cells (MC), erythrocytes (RBC) and plasma. The expression level of pre-miR-488 in each fraction is shown in Figure 2D. The expression of pre-miR-488 in plasma was evaluated as follows: [putative plasma] $=[$ whole blood $]-[\mathrm{PMN}]-[\mathrm{MC}]-$ $[\mathrm{RBC}]$. This approach was necessary because quantitative analysis of plasma RNA could not be performed because of the low levels present, although pre-miR-488 was detected in plasma by traditional RT-PCR (Figure 2E). The median relative ratios of $\mathrm{RBC}, \mathrm{MC}, \mathrm{PMN}$ and putative plasma to whole blood were $0.022,0.000,0.009$ and 0.965 , respectively. Pre-miR-488 expression was estimated to be much higher in plasma than in polymorphonuclear leukocytes, mononuclear cells, and erythrocytes. This finding indicates that pre-miR488 is localized mainly in plasma in blood.

Correlation between amounts of pre-miR-488 and $\mathrm{miR}-488$ $5 p$ in blood. We randomly chose 27 breast cancer cases from which we obtained high-quality RNA and examined the correlation between the amounts of pre-miR-488 and miR488-5p in blood. Spearman's rank correlation co-efficient demonstrated a significantly positive correlation between
pre-miR-488 expression and miR-488-5p levels in blood $(\mathrm{r}=0.457, p=0.017)$ (Figure $2 \mathrm{~F})$.

Prognostic significance of the amount of pre-miR-488 in blood. The prognostic significance of pre-miR-488 expression in blood was assessed using 330 IDC cases who underwent curative surgery for OS and RFS. Cases were divided into two groups as described in Materials and Methods. The cutoff value was 0.052 . High pre-miR-488 expression was not significantly associated with poor OS $(p=0.055)$, but with poor RFS $(p=0.043)$ (Figure 3$)$. Then, univariate and multivariate analyses for RFS were performed (Table I). Univariate analysis showed that lymphatic involvement, lymph node metastasis, PgR and high levels of pre-miR-488 were statistically significant prognostic factors for RFS ( $p=0.0005,0.002,0.028$ and 0.032 , respectively). Pre-miR488 expression and clinicopathological factors such as tumor size, lymph node metastasis, PgR, and HER2 were included in the multivariate analysis. Notably, multivariate analysis demonstrated that pre-miR-488 expression in blood was an independent prognostic factor for RFS in patients with IDC $(\mathrm{HR}=3.19, p=0.030)$. Moreover, subgroup analyses for RFS were performed according to each subtype. As shown in Figure 3C, the high pre-miR-488 expression group exhibited poorer prognosis only in the HER2-positive group $(p=0.039)$.

Clinicopathological significance of pre-miR-488 expression in blood. The relationship between clinicopathological factors and pre-miR-488 expression in blood was examined using the 330 patients with IDC. As shown in Table II, no significant difference was found between the high and low pre-miR-488 expression groups regarding clinicopathological factors such as age, tumor size, nuclear grade, venous involvement, lymphatic involvement, lymph node metastasis, the status of ER, PgR and HER2 and subtype. 
Table II. Relationship between clinicopathological factors and pre-miR488 expression in blood in breast cancer.

\begin{tabular}{|c|c|c|c|}
\hline Variables & $\begin{array}{c}\text { Low }(\mathrm{n}=129) \\
\text { Number }(\%)\end{array}$ & $\begin{array}{l}\text { High }(\mathrm{n}=201) \\
\text { Number }(\%)\end{array}$ & $p$-Value \\
\hline Age (years) & & & 0.409 \\
\hline$<65$ & $97(75.2)$ & $150(74.6)$ & \\
\hline$\geq 65$ & $24(18.6)$ & $48(23.9)$ & \\
\hline Unknown & $8(6.2)$ & $3(1.5)$ & \\
\hline Pathological tumor size & & & 0.496 \\
\hline 1 & $65(50.4)$ & $108(53.7)$ & \\
\hline 2,3 & $64(49.6)$ & $89(44.3)$ & \\
\hline Unknown & $0(0.0)$ & $4(2.0)$ & \\
\hline Nuclear grade & & & 0.136 \\
\hline 1,2 & $77(59.7)$ & $137(68.2)$ & \\
\hline 3 & $44(34.1)$ & $54(26.9)$ & \\
\hline Unknown & $8(6.2)$ & $10(5.0)$ & \\
\hline Venous involvement & & & 0.812 \\
\hline$(-)$ & $118(91.5)$ & $184(91.5)$ & \\
\hline$(+)$ & $8(6.2)$ & $11(5.5)$ & \\
\hline Unknown & $3(2.3)$ & $6(3.0)$ & \\
\hline Lymphatic involvement & & & 0.724 \\
\hline$(-)$ & $78(60.5)$ & $125(62.2)$ & \\
\hline$(+)$ & $49(38.0)$ & $71(35.3)$ & \\
\hline Unknown & $2(1.6)$ & $5(2.5)$ & \\
\hline Lymph node metastasis & & & 0.816 \\
\hline$(-)$ & $83(64.3)$ & $126(62.7)$ & \\
\hline$(+)$ & $46(35.7)$ & $74(36.8)$ & \\
\hline Unknown & $0(0.0)$ & $1(0.5)$ & \\
\hline ER & & & 0.797 \\
\hline$(-)$ & $32(24.8)$ & $52(25.9)$ & \\
\hline$(+)$ & $97(75.2)$ & $146(72.6)$ & \\
\hline Unknown & $0(0.0)$ & $3(1.5)$ & \\
\hline PgR & & & 0.732 \\
\hline$(-)$ & $55(42.6)$ & $81(40.3)$ & \\
\hline$(+)$ & $74(57.4)$ & $118(58.7)$ & \\
\hline Unknown & $0(0.0)$ & $2(1.0)$ & \\
\hline HER2 & & & 0.321 \\
\hline$-(0,1)$ & $86(66.7)$ & $118(58.7)$ & \\
\hline$+(2,3)$ & $36(27.9)$ & $64(31.8)$ & \\
\hline Unknown & $7(5.4)$ & $19(9.5)$ & \\
\hline Subtype & & & 0.490 \\
\hline HR+/HER2- & $70(54.3)$ & $100(49.8)$ & \\
\hline $\mathrm{HR} \pm / \mathrm{HER} 2+$ & $36(27.9)$ & $64(31.8)$ & \\
\hline $\mathrm{TN}$ & $16(12.4)$ & $18(9.0)$ & \\
\hline Unknown & $7(5.4)$ & $19(9.5)$ & \\
\hline
\end{tabular}

The correlation was analyzed by the Fisher's exact test. HR: Hormone receptor; $\mathrm{TN}$ : triple negative.

\section{Discussion}

In this study of breast cancer, it was demonstrated that high expression of pre-miR-488 in blood was an independent biomarker that predicted recurrence of disease. Furthermore, pre-miR-488 expression was positively correlated with miR488-5p levels in blood, which was shown to be a recurrencerelated $\mathrm{miR}$, by $\mathrm{miR}$ array analysis. To the best of our knowledge, this is the first study to explore pre-miR as a clinical biomarker in cancer patients and determine the clinical relevance of circulating pre-miR-488 in breast cancer.

Numerous reports have been published on the utility of miRs as diagnostic or prognostic biomarkers in malignancies. However, the expression analysis of mature miR requires high-quality RNA and specific primers for RT and PCR assessment. Moreover, an accurate internal control for mature miR is still controversial, although researchers have attempted to identify reliable and reproducible markers (6). These issues may lead to incorrect conclusions about the clinical significance of $\mathrm{miR}$ expression. On the other hand, expression analysis of pre-miR can be performed by conventional methods using universal materials. Focusing on pre-miR may allow laboratories to overcome the problems of miR expression analysis for use as biomarkers.

High expression of pre-miR-488 in blood was associated with a poor prognosis in breast cancer patients, especially in HER2-positive cases, although pre-miR-488 was downregulated in the blood and tumor tissues of breast cancer patients compared with healthy controls. So far, miR-488 is reported to act as a tumor suppressor. It is down-regulated in a range of tumors, including pancreatic cancer (17), prostate cancer (18), hepatocellular carcinoma (19), ovarian cancer (20), gastric cancer (21), colorectal cancer (22), non-small cell lung cancer $(23,24)$, melanoma $(25)$ and gastrointestinal stromal tumor (26) where it targets $J A K 1, A R, A D A M 9$, Six 1, PAX6, PHF8, elF3a or PRKDC, respectively. Pre-miR-488 was also found to be down-regulated in breast tumor tissues compared with normal controls. This observation suggests that miR-488 may also act as a tumor suppressor in breast cancer tissue. Unexpectedly, a survival analysis using blood demonstrated that high expression of circulating pre-miR488 was a poor prognostic biomarker in breast cancer.

Circulating pre-miR-488 was mainly located in the plasma. miRs are packaged inside exosomes (extracellular vesicles) that are secreted into the blood. Exosomal miRs are probably delivered to recipient cells where they regulate target gene expression. Pre-miRs are also enveloped by exosomes in body fluids and function as intercellular messengers $(12,13)$. We observed that the high expression of pre-miR-488 in blood was associated with a poor prognosis in breast cancer, especially in HER2-positive cases, although pre-miR-488 was downregulated in the blood of breast cancer patients. These results imply that circulating pre-miR-488 plays an oncogenic role as an intercellular messenger, as does miR-203 in CRC as we reported previously (5). Circulating pre-miR-488 might affect the HER2 signal pathway because its high expression was associated with poor prognosis only in HER2-positive patients.

Collectively, miR-488 may have the potential to work as a tumor suppressor in tumor tissues, whereas as an oncogene in blood. Recent studies have focused on the contrasting roles of miRs in oncogenesis and tumor suppression (27). We speculate that pre-miR-488 may have dual roles in breast 
cancer development. Namely, it may function as a tumor suppressor during tumorigenesis and an enhancer during tumor progression as is the case with DNA damage response genes $(28,29)$. Furthermore, miR may have site-dependent functions that promote tumor development (5). miR localization should be taken into account when determining its clinical significance. Further examination of miR-488 to define its function and significance as a biomarker in various sites in breast cancer patients will be required for the proper clinical interpretation.

In summary, pre-miR-488 expression in blood could be a novel prognostic biomarker for predicting recurrence in breast cancer patients.

\section{Conflicts of Interest}

The Authors have no conflicts of interest to disclose.

\section{Acknowledgments}

The Authors thank Dr. Tyler Lahusen for helpful comments and English proofreading. The Authors also thank K. Oda, M. Kasagi, S. Sakuma, N. Mishima and T. Kawano for their excellent technical assistance. This work was supported in part by the following grants and foundations: Japan Society for the Promotion of Science (JSPS) Grant-in-Aid for Science Research (Grant Numbers JP16K07177, JP16K10543, JP16K19197, JP17K16454, JP17K16521, JP17K10593 and JP17K19608); OITA Cancer Research Foundation; Daiwa Securities Health Foundation; Grant-in-Aid for Scientific Research on Innovative Areas (15H0912); Priority Issue on Post-K computer (hp170227) (hp170227, hp160219); JSPS KAKENHI (15H05707); Eli Lilly Japan K.K. Grant; Japanese Foundation for Multidisciplinary Treatment of Cancer.

\section{References}

1 Jameson JL and Longo DL: Precision medicine--personalized, problematic, and promising. N Engl J Med 372: 2229-2234, 2015.

2 Di Meo A, Bartlett J, Cheng Y, Pasic MD and Yousef GM: Liquid biopsy: a step forward towards precision medicine in urologic malignancies. Mol Cancer 16: 80, 2017.

3 Shimomura A, Shiino S, Kawauchi J, Takizawa S, Sakamoto H, Matsuzaki J, Ono M, Takeshita F, Niida S, Shimizu C, Fujiwara Y, Kinoshita T, Tamura K and Ochiya T: Novel combination of serum microRNA for detecting breast cancer in the early stage. Cancer Sci 107: 326-334, 2016.

4 Gallo A, Tandon M, Alevizos I and Illei GG: The majority of microRNAs detectable in serum and saliva is concentrated in exosomes. PLoS One 7: e30679, 2012.

5 Takano Y, Masuda T, Inuma H, Yamaguchi R, Sato K, Tobo T, Hirata H, Kuroda Y, Nambara S, Hayashi N, Iguchi T, Ito S, Eguchi H, Ochiya T, Yanaga K, Miyano S and Mimori K: Circulating exosomal microRNA-203 is associated with metastasis possibly via inducing tumor-associated macrophages in colorectal cancer. Oncotarget 8: 78598-78613, 2017.

6 Masuda T, Hayashi N, Kuroda Y, Ito S, Eguchi H and Mimori $\mathrm{K}$ : MicroRNAs as biomarkers in colorectal cancer. Cancers (Basel) 9: 124, 2017.
7 Lin S and Gregory RI: MicroRNA biogenesis pathways in cancer. Nat Rev Cancer 15: 321-333, 2015.

8 White NM, Fatoohi E, Metias M, Jung K, Stephan C and Yousef GM: Metastamirs: a stepping stone towards improved cancer management. Nat Rev Clin Oncol 8: 75-84, 2011.

9 Shinden Y, Akiyoshi S, Ueo H, Nambara S, Saito T, Komatsu H, Ueda M, Hirata H, Sakimura S, Uchi R, Takano Y, Iguchi T, Eguchi H, Sugimachi K, Kijima Y, Ueo H, Natsugoe S and Mimori K: Diminished expression of MiR-15a is an independent prognostic marker for breast cancer cases. Anticancer Res 35: 123-127, 2015.

10 Matsumura T, Sugimachi K, Iinuma H, Takahashi Y, Kurashige J, Sawada G, Ueda M, Uchi R, Ueo H, Takano Y, Shinden Y, Eguchi $\mathrm{H}$, Yamamoto H, Doki Y, Mori M, Ochiya T and Mimori K: Exosomal microRNA in serum is a novel biomarker of recurrence in human colorectal cancer. Br J Cancer 113: 275-281, 2015.

11 Tarver JE, Sperling EA, Nailor A, Heimberg AM, Robinson JM, King BL, Pisani D, Donoghue PC and Peterson KJ: miRNAs: small genes with big potential in metazoan phylogenetics. Mol Biol Evol 30: 2369-2382, 2013.

12 Gu Y, Li M, Wang T, Liang Y, Zhong Z, Wang X, Zhou Q, Chen L, Lang Q, He Z, Chen X, Gong J, Gao X, Li X and Lv X: Lactation-related microRNA expression profiles of porcine breast milk exosomes. PLoS One 7: e43691, 2012.

13 Chen TS, Lai RC, Lee MM, Choo AB, Lee CN and Lim SK: Mesenchymal stem cell secretes microparticles enriched in premicroRNAs. Nucleic Acids Res 38: 215-224, 2010.

14 Ueo H, Sugimachi K, Gorges TM, Bartkowiak K, Yokobori T, Muller V, Shinden Y, Ueda M, Ueo H, Mori M, Kuwano H, Maehara Y, Ohno S, Pantel K and Mimori K: Circulating tumour cell-derived plastin3 is a novel marker for predicting long-term prognosis in patients with breast cancer. Br J Cancer 112: 15191526, 2015.

15 Coates AS, Winer EP, Goldhirsch A, Gelber RD, Gnant M, Piccart-Gebhart M, Thurlimann B, Senn HJ and Panel M: Tailoring therapies--improving the management of early breast cancer: St Gallen International Expert Consensus on the Primary Therapy of Early Breast Cancer 2015. Ann Oncol 26: 15331546, 2015.

16 Mizuno H, Kitada K, Nakai K and Sarai A: PrognoScan: a new database for meta-analysis of the prognostic value of genes. BMC Med Genomics 2: 18, 2009.

17 Yu DL, Zhang T, Wu K, Li Y, Wang J, Chen J, Li XQ, Peng XG, Wang JN and Tan LG: MicroRNA-448 suppresses metastasis of pancreatic ductal adenocarcinoma through targeting JAK1/STAT3 pathway. Oncol Rep 38: 1075-1082, 2017.

18 Sikand K, Slaibi JE, Singh R, Slane SD and Shukla GC: miR 488* inhibits androgen receptor expression in prostate carcinoma cells. Int J Cancer 129: 810-819, 2011.

$19 \mathrm{Hu}$ D, Shen D, Zhang M, Jiang N, Sun F, Yuan S and Wan K: MiR-488 suppresses cell proliferation and invasion by targeting ADAM9 and lncRNA HULC in hepatocellular carcinoma. Am J Cancer Res 7: 2070-2080, 2017.

20 Yang Z, Feng Z, Gu J, Li X, Dong Q, Liu K, Li Y and OuYang L: microRNA-488 inhibits chemoresistance of ovarian cancer cells by targeting Six 1 and mitochondrial function. Oncotarget 8: 80981-80993, 2017.

21 Zhao Y, Lu G, Ke X, Lu X, Wang X, Li H, Ren M and He S: miR-488 acts as a tumor suppressor gene in gastric cancer. Tumour Biol 37: 8691-8698, 2016. 
22 Lv Y, Shi Y, Han Q and Dai G: Histone demethylase PHF8 accelerates the progression of colorectal cancer and can be regulated by miR-488 in vitro. Mol Med Rep 16: 4437-4444, 2017.

23 Fang C, Chen YX, Wu NY, Yin JY, Li XP, Huang HS, Zhang W, Zhou HH and Liu ZQ: MiR-488 inhibits proliferation and cisplatin sensibility in non-small-cell lung cancer (NSCLC) cells by activating the eIF3a-mediated NER signaling pathway. Sci Rep 7: 40384, 2017.

24 Patnaik SK, Kannisto E, Knudsen S and Yendamuri S: Evaluation of microRNA expression profiles that may predict recurrence of localized stage I non-small cell lung cancer after surgical resection. Cancer Res 70: 36-45, 2010.

25 Li N, Ma Y, Ma L, Guan Y, Ma L and Yang D: MicroRNA-488$3 p$ sensitizes malignant melanoma cells to cisplatin by targeting PRKDC. Cell Biol Int 41: 622-629, 2017.

26 Tong HX, Zhou YH, Hou YY, Zhang Y, Huang Y, Xie B, Wang JY, Jiang Q, He JY, Shao YB, Han WM, Tan RY, Zhu J and Lu WQ: Expression profile of microRNAs in gastrointestinal stromal tumors revealed by high throughput quantitative RT-PCR microarray. World J Gastroenterol 21: 5843-5855, 2015.
27 Svoronos AA, Engelman DM and Slack FJ: OncomiR or Tumor Suppressor? The Duplicity of MicroRNAs in Cancer. Cancer Res 76: 3666-3670, 2016.

28 Jackson SP and Bartek J: The DNA-damage response in human biology and disease. Nature 461: 1071-1078, 2009.

29 Tian H, Gao Z, Li H, Zhang B, Wang G, Zhang Q, Pei D and Zheng J: DNA damage response--a double-edged sword in cancer prevention and cancer therapy. Cancer Lett 358: 8-16, 2015.

Received June 6, 2018

Revised June 14, 2018

Accepted June 15, 2018 